\title{
PERÚ: MAPA DE VIOLENCIA FAMILIAR, A NIVEL DEPARTAMENTAL, SEGÚN LA ENDES 2007-2008. CARACTERÍSTICAS E IMPLICANCIAS
}

\author{
PERU: MAP OF FAMILY VIOLENCE, AT DEPARTAMENTAL LEVEL ACCORDING \\ TO ENDES 2007-2008: FEATURES AND IMPLICATIONS \\ Manuel Miljanovich C. 1; Violeta Nolberto S.; Martha Martina Ch.; Rosa Elena Huerta R.; \\ Socorro Torres V.; Fernando Camones G. \\ Universidad Nacional Mayor de San Marcos, Perú \\ (RECIBIDO EL 12/10/2010, ACEPTADO EL 2/12/2010)
}

\begin{abstract}
RESUMEN
El objetivo central del presente estudio fue elaborar un Mapa de la Violencia Familiar en el Perú a nivel nacional y departamental, a partir de los resultados de la Encuesta Demográfica y de Salud Familiar ENDES 2007-2008. Se elaboró un Índice Global de Violencia Familiar (IGVF) que sintetiza los indicadores de Violencia Física, Violencia Psicológica y Violencia Sexual. La construcción de tal índice se efectúo mediante el método multivariante PRINCALS, llamado también Análisis de componentes principales categóricos o no lineales. Dado que las variables están medidas en escalas nominal u ordinal, no están relacionadas de un modo lineal $\mathrm{y}$, por tanto, se requiere emplear el procedimiento generalmente denominado escalamiento óptimo, empleando el citado método. Dicho índice permitió trazar el mapa en referencia, cuya principal ventaja es superar la información fragmentaria sobre violencia física, psicológica, sexual y algunas variables asociadas, tales como edad, estado civil, grado educativo y nivel socioeconómico. La estimación del índice Global de Violencia Familiar (IGVF), a nivel nacional, es igual a 12,58 y el índice de Violencia Psicológica (IVP) es igual a 23,40, ambos con coeficiente de variación bajo $(0,014)$. Los departamentos de Loreto, Apurímac, Pasco, Madre de Dios y Arequipa, constituyen el 20\% con mayor IGVF, el mismo que fluctúa entre 14,28 y 16,13. El $20 \%$ de departamentos con IGVF más bajo, cuyos valores fluctúan entre 10,43 y 13,38, está integrado por Ica, Lima, Cajamarca y Lambayeque. La conclusión más importante es que en el Perú de hoy, la violencia familiar, en sus diferentes formas, violencia física, violencia psicológica y violencia sexual, está extendida y arraigada en todo el territorio y distribuida con ciertas diferencias a nivel departamental.
\end{abstract}

Palabras clave: Violencia familiar, mapa nacional y departamental.

\begin{abstract}
The main objective of this study was to develop a map of Domestic Violence in Peru at the national and departmental level, based on the results of the Population and Family Health ENDES 2007-2008. Developed a Global Index Family Violence (IGVF) that summarizes the indicators of physical violence, psychological and sexual violence. The construction of
\end{abstract}

1 Profesor Principal de la Facultad de Psicología de la UNMSM. E-mail: civonajlim@gmail.com 
such an index was performed by multivariate PRINCALS method, also called categorical principal component analysis or nonlinear. Since the variables are measured in nominal or ordinal scales are not related in a linear fashion and, therefore, is required to use the optimal scaling procedure is usually called, using the above method. This index allowed the mapping in question, whose main advantage is to overcome the fragmentary information on physical, psychological, sexual and associated variables such as age, marital status, educational level and socioeconomic status. The estimate of the Global Family Violence (IGVF), nationally, is equal to 12,58 and the rate of psychological violence (IVP), is equal to 23,40 , both with low coefficient of variation $(0,014)$. The departments of Loreto, Apurimac, Pasco, Mother of God and Arequipa, up 20\% more IGVF, it fluctuates between 14,28 and 16,13. 20\% of departments IGVF lower, with values ranging between 10,43 and 13,38, consists of Ica, Lima, Cajamarca and Lambayeque. The most important conclusion is that in Peru today, domestic violence in its different forms, physical violence, psychological violence and sexual violence is widespread and entrenched throughout and distributed with some differences at the departmental level.

Keywords: Domestic violence, national and district map.

\section{INTRODUCCIÓN}

La violencia doméstica constituye un problema de salud pública, que no obstante ser frecuente sigue encubierto afectando a las mujeres independientemente de la edad, raza, cultura y nivel socioeconómico. La violencia familiar es parte de la agenda política de los países y en este caso el sector académico presta especial atención a esta temática que no solo afecta a la mujer como persona individual, sino que detrás de la violencia contra una mujer, existe violencia en el seno de la familia y por lo tanto, la sociedad está involucrada en este problema.

El Plan Nacional contra la Violencia en la Mujer 2009-2015 del Programa Nacional Contra la Violencia Familiar y Sexual (PNCVFS) del Ministerio de la Mujer y Desarrollo Familiar (MIMDES), señala que la violencia es un fenómeno de alta complejidad, de naturaleza multicausal y expresión multidimensional, que afecta tanto a los individuos como a las sociedades. Constituye un problema de suma gravedad que involucra a la mayoría de la población, e impide la construcción de relaciones democráticas al interior de una sociedad y entraña el riesgo del desborde social.

En el caso peruano, el reciente estudio multicéntrico de la OMS (2005) confirma que su hogar, el lugar supuestamente más seguro y de mayor protección para la mujer, el ámbito que mayor riesgo representa para su integridad física y sexual; y que su principal agresor es la persona con quien está unida por lazos afectivos y económicos y que además comparten un proyecto común de vida.

El Informe Mundial (2002) elaborado por la OMS establece que la violencia debe afrontarse desde la perspectiva de salud pública, y las intervenciones se deben hacer en los grupos que corren mayor riesgo de enfermedades o lesiones. Mercy J.A. et al. (1993) establecen que el proceso de salud pública aborda cualquier amenaza para el bienestar y consta de las cuatro etapas siguientes: definir y observar la magnitud del problema; identificar sus causas; formular y poner a prueba modos de afrontarlo; y aplicar ampliamente las medidas de eficacia probada, con estrategia multidisciplinar. Uno 
de los tipos de violencia preocupante es la violencia familiar, porque se produce entre miembros de la familia o compañeros sentimentales, y suele ocurrir en el hogar, incluso está legislado en la Ley de Protección contra la Violencia Familiar en nuestro país.

El Centro de Información de las Naciones Unidas (2007-2012) señala que "en algún momento de sus vidas, más de la mitad de todas las mujeres latinoamericanas han sido objeto de agresiones en sus hogares. Un 33\% fueron víctimas de abusos sexuales entre los 16 y 49 años, mientras el $45 \%$ recibió amenazas, insultos y la destrucción de objetos personales".

Klevens Joanne (2001) estudió 3157 mujeres que convivían con un compañero íntimo, el $26,5 \%$ informó que había sido abofeteada o empujada por su compañero actual (violencia física) y $13,3 \%$ que había sido agredida gravemente por medio de golpes con el puño cerrado, patadas, golpes con algún objeto, "golpizas" y amenazas con un cuchillo o arma de fuego (violencia física). El 26,2\% de ellas indicaron que su compañero les imponía alguna prohibición (violencia psicológica). Es un problema complejo, enraizado en la interacción de ellos; algunos factores de riesgo pueden ser privativos de un tipo determinado de violencia, pero es más frecuente que los diversos tipos de violencia compartan varios factores de riesgo. La frecuencia del conflicto con la pareja y las prohibiciones fueron los factores más fuertemente asociados con la violencia, según el análisis multivariado.

Bardales y Huallpa (2005) indican que a nivel nacional 1 de cada 10 mujeres (entre $15 \mathrm{y}$ 49 años) alguna vez ha sufrido en el último año violencia física por parte de su pareja, así como ha sido forzada a tener relaciones sexuales; 1 de cada 33 varones actualmente sufrió violencia física en el último año por parte de su pareja en los distritos de Tarapoto, Puno y San Juan de Lurigancho; y 8 de cada 10 niños, niñas y adolescentes refieren maltrato en el hogar en el último año en los distritos de Cusco, Iquitos y San Martín.

Miguel Ramos (2003) señala que la violencia contra las mujeres por parte de la pareja es un problema social generalizado, con altísimas prevalencias tanto en Lima Metropolitana como en el departamento de Cusco, las cuales se distribuyen de manera diferencial según edad. Son las mujeres más jóvenes las que sufren con mayor fuerza tales actos violatorios a sus derechos humanos. Este problema demanda la realización de estudios más específicos que permitan comprender mejor la experiencia conyugal o de pareja a edades más tempranas, y su relación con la violencia. La prevalencia de violencia física o sexual ocurrida durante toda la vida de pareja nos señala claramente que una mayoría de varones adultos en Lima Metropolitana (51\%) y en el departamento de Cusco $(69 \%)$, ejerce violencia contra sus parejas (Güezmes, Palomino y Ramos, 2002). Esto demuestra que no se trata de un problema aislado de unos cuantos enfermos mentales, alcohólicos o drogadictos, sino que estamos ante un fenómeno social: vivimos en una cultura que justifica la violencia contra la mujer y una institucionalidad que la permite.

Viviano (2007) sostiene que la violencia familiar, como la sexual, es un problema grave y frecuente de proporciones epidémicas, que afecta sistemáticamente a importantes sectores de la población, especialmente mujeres, niñas, niños y personas adultas mayores y que las acciones para combatirla deben constituirse en eje básico de la agenda local de desarrollo. 
El Programa Nacional contra la Violencia Familiar y Sexual (PNCVFS) durante el año 2006 atendió a un total de 457 personas afectadas por violencia familiar y sexual a través del Centro Emergencia Mujer (CEM), Iquitos; de los cuales el 86,2\% fueron mujeres; el $57,5 \%$ manifestaron ser víctimas de violencia psicológica, el 20,1\% fueron por violencia física y el 22,3\%, por violencia sexual. Según cifras reportadas por Bardales, et al. (2006), en los CEM de Lima durante el año 2007, el 80,1\% de casos atendidos fueron por violencia física y/o psicológica, y el 19,9\% por violencia sexual. MIMDES, a septiembre de 2008, ha registrado en los CEM de Lima 11397 casos de violencia familiar, de los cuales el 77,34\% correspondieron a Lima Metropolitana, el 15,03\% al Callao y el resto, a Lima Provincias. Un total de 28 mil 300 denuncias por violencia familiar y sexual se registraron en todo el país.

El PNCVFS reporta que se han atendido 39820 casos de violencia familiar en los CEM a nivel nacional, hasta noviembre del 2010. Según el Ministerio del Interior, durante el primer semestre del 2009 se registraron 48,602 denuncias por violencia familiar en todo el país, de las cuales $5156(10,61 \%)$ fueron contra varones y 43,446 $(89,39 \%)$ contra mujeres. El 83,03\% $(36,077)$ de denuncias fueron efectuadas por mujeres de 18 a 50 años de edad. Los departamentos que registran mayores denuncias son: Lima 43,08\% (20,940 casos), Arequipa 11,87\% (5770 casos) y Cusco 6,15\% (2991 casos).

En un estudio cualitativo de la violencia familiar, durante el período 2008-2009, (Miljanovich et al., 2010) se identificaron modelos explicativos de la génesis y evolución de la violencia familiar en zonas marginales con pobreza crítica y extrema. Esta investigación pone de relieve el deterioro de la personalidad de la mujer victimizada y de la dinámica familiar, así como la repercusión de la violencia familiar en el rendimiento escolar de niños y adolescentes, en términos de repitencia, deserción y desmotivación.

La mayoría de las investigaciones sobre violencia familiar en el país son sólo descriptivas y con ámbitos restringidos cuyos resultados no favorecen una cabal comprensión de la violencia familiar en forma integral y menos a nivel nacional. Por otro lado, al contar con información resultante de encuestas a nivel nacional se abre un espacio de posibilidades de análisis e interpretación de la temática de la violencia relacionándola con otras variables, con miras a proponer algunas medidas preventivas y de protección a la mujer y, por ende, a la familia en general. Por lo tanto, se hace necesaria la realización de una investigación que empleando información estadística a nivel nacional (ENDES 2008), nos muestre la distribución de la violencia a través de un mapa a nivel nacional y que favorezca la generación y aplicación de modelos explicativos de la violencia familiar.

Las metas del nuevo milenio en salud planteadas en septiembre de 2000 por la OMS es todo un reto que deben alcanzar los estados. En la llamada Cumbre del Milenio de las Naciones Unidas, los líderes mundiales de 189 estados adoptaron un conjunto de objetivos y metas cuantificables y delimitadas en tiempo para combatir la pobreza, el hambre, las enfermedades, el analfabetismo, la degradación del medio ambiente, la discriminación contra la mujer, y comprometiéndose con los principios de los derechos humanos, la gobernanza responsable y la democracia. Se esbozaron en la Declaración del Milenio, y desde entonces se han resumido y operacionalizado a través de un paquete de 8 objetivos generales, 18 metas y 48 indicadores, comúnmente denominados los Objetivos de 
Desarrollo del Milenio (ODM) a alcanzarse para el año 2015 (OMS, 2004). Justamente, el tercer objetivo, "Promover la igualdad entre los géneros y la autonomía de la mujer", está relacionado con el presente estudio, por ello, a fin de focalizar la violencia familiar a nivel departamental en nuestro país, se planteó como objetivo fundamental del presente estudio la elaboración de un MAPA DE VIOLENCIA FAMILIAR a nivel nacional y departamental a partir de los resultados de la Encuesta Demográfica y de Salud Familiar (ENDES) 2007-2008.

\section{MÉTODO}

Los mapas de pobreza son instrumentos útiles para la focalización del gasto destinado a la lucha contra la pobreza y otros males en países como el nuestro, típicamente caracterizados por presentar severos problemas de pobreza y limitaciones en el presupuesto disponible para combatirla.

La focalización geográfica tiene como objetivo la identificación y atención de áreas pobres y toma en cuenta las diferencias significativas en los niveles de vida observados entre áreas geográficas. Javier Escobal et al. (2001) mencionan que diversos estudios sobre el tema, como el de Ravallion y Woodon (1997) o el de Bigman y Fofack (2000), sostienen que las diferencias observadas entre los niveles de vida promedio de diferentes comunidades parecerían ser demasiado grandes para ser explicadas por diferencias en características individuales o familiares observables, no geográficas y móviles (variables demográficas, laborales, educacionales, etc.) y que por consiguiente es importante incluir características geográficas del tipo en cuestión.

La fuente de datos utilizada en este estudio es secundaria. Se trata de la ENDES CONTINUA, que es una investigación que realiza el INEI (Instituto Nacional de Estadística e Informática) en el marco del Programa Mundial de las Encuestas de Demografía y Salud para obtener información actualizada y efectuar análisis del cambio, tendencias y determinantes de la fecundidad, mortalidad y la salud en el Perú. La población bajo estudio está integrada por mujeres en edad fértil y sus hijos menores de 5 años. Es una investigación transversal.

La ENDES para lograr sus objetivos considera una muestra probabilística de áreas, estratificada, bietápica y autoponderada a nivel departamental, por área de residencia: Urbana y Rural. El marco de la ENDES es un Marco Maestro de áreas que es elaborada con información de los Censos Nacionales de Población y Vivienda del 2007. En el área urbana las Unidades de Muestreo son: el Conglomerado y la Vivienda Particular. En el área rural las unidades de muestreo son: el área de Empadronamiento Rural y la Vivienda Particular.

La muestra está constituida por 1001 conglomerados, distribuidos proporcionalmente en el área urbana y rural de cada departamento. El número de conglomerados por departamento es de 37, con excepción de Lima, donde se seleccionaron 112 conglomerados. El número de hogares es de 27 204, de los cuales 25633 fueron objeto de entrevistas. En los hogares visitados, se encontraron un total de 23094 mujeres elegibles de 15 a 49 años de edad y de las cuales se obtuvo un total de 22558 entrevistas completas. La muestra seleccionada representa la totalidad de la población. 
La base de datos del módulo Violencia Familiar de la ENDES 2007-2008 está constituida por una matriz de datos, en la que las filas están formadas por las Objetos/Sujeto. Mujeres y las columnas, por variables cualitativas medidas bajo escala nominal $u$ ordinal, esto es, los datos están considerados como categorías (atributos) o niveles.

Para la elaboración del Mapa se consideraron los datos de la Sección 10. Violencia Doméstica de la ENDES Continua 2007-2008. Se les preguntó en forma directa a las mujeres que habían estado alguna vez casadas o unidas, acerca de si su última pareja (esposo o compañero) había ejercido sobre ellas violencia verbal o física. Asimismo, para contextualizar la violencia contra la mujer, se consideraron datos de la Sección 1. Antecedentes de la entrevistada; Sección 2: Reproducción y la Sección 5: Nupcialidad.

El total de mujeres que habían estado alguna vez casadas o unidas es igual a 22558 y de cada una de ellas se registraron datos de 23 variables; lo que con una tabla de frecuencias a nivel de una variable o de dos variables no brinda información relevante; por tanto, se empleó una técnica multivariante para sintetizar la información sobre violencia doméstica de cada mujer, es decir construir un índice, que es todo aquello que nos da un indicio o señal de algo. En estadística, este índice por lo general es de naturaleza numérica, que cuantifica algo (violencia doméstica) que se quiere medir, ya sea de carácter unidimensional o multidimensional. Para el trabajo realizado este índice es de carácter multidimensional, porque se refiere a numerosas variables. Se construyó un índice global de la violencia que sintetiza 23 variables.

Una técnica multivariante que sintetiza variables es el Análisis de Componentes Principales (ACP) (Mardia K. V.; Kent J. T.; Bibby J. M., 1979), que reduce la dimensión de un conjunto de datos, de variables con alta correlación lineal, en otro conjunto más pequeño de componentes (nuevas variables) no correlacionadas, cuando las variables se han medido por lo menos en escala de intervalos, es decir se están analizando datos de variables cuantitativas.

Bell (2004) explica que al asignar valores a las categorías o niveles de una variable categórica generalmente se considera la medición o cuantificación de manera equidistante, lo que es erróneo. Por ello, se deben transformar a las variables categóricas asignándole cuantificaciones numéricas a sus categorías o niveles, lo que se conoce como "valores óptimos". Empleando esta definición Young, Takane y de Leeuw (1978) desarrollaron programas y algoritmos para cuantificar datos cualitativos, llamado Programa ALSOS (Alternating Least Squares Optimal), Mínimos Cuadrados Alternativos con Escalamiento Óptimo, que tienen por objetivo determinar los valores para las categorías que maximizan la calidad del modelo ajustado, llamados "valores óptimos".

Forrest W. Young (1981) señala que los "valores óptimos" maximizan la eficiencia del modelo. El algoritmo PRINCALS (Análisis de Componentes Principales no Lineal) es una técnica que permite asignar valores óptimos y luego aplicar el ACP para caracterizar o analizar la estructura de los datos y reducir la dimensión.

Estos valores óptimos poseen propiedades métricas como lo indican Meulmann y Heiser (2001), y por tanto el análisis de datos categóricos recogidos en encuestas con estructura no lineal, muy bien son analizados mediante ACPNL o PRINCALS. 
Sin embargo, en las encuestas es común medir variables bajo escala nominal u ordinal, como en este caso, por tanto, el supuesto de linealidad entre pares de variables (ítems) es nulo; en este caso, el ACP no es una buena herramienta para analizar datos que no son lineales, pero el Análisis de Componentes Principales no Lineal (ACPNL) es una buena alternativa para analizar situaciones de este tipo. Para lo cual es necesario transformar las variables originales asignando valores a sus categorías o niveles y luego correlacionándolas para caracterizar o analizar la estructura de datos, en términos de "valores óptimos".

Entonces, la técnica multivariante utilizada para la construcción de los índices globales de violencia familiar es la técnica multivariada conocida como "Análisis de Componentes Principales Categóricos o No lineales" (PRINCALS), que tiene como objetivo obtener nuevas variables llamadas FACTORES, donde cada uno de ellos explica el porcentaje de variabilidad total (inercias). Estos FACTORES están correlacionados con las variables originales (ítems), y la consistencia interna de cada factor es medida por el estadístico Alpha de Cronbach, el cual nos indica la correlación conjunta que existe entre las variables contenidas en un factor en particular.

Esta técnica multivariada permite reducir las dimensiones originalmente consideradas, lo que lleva a la construcción de otras nuevas variables, que son en número mucho menor a las originales y que se encuentran incorrelacionadas. Por tanto, el aporte de cada una de estas nuevas variables al conocimiento del fenómeno en estudio, es evidente, logrando con esto eliminar la redundancia de información.

El proceso que sigue PRINCALS para la estimación de los parámetros del modelo es el procedimiento conocido como Alternating Least Squares o alternancia de mínimos cuadrados (ALSOS). La estimación de mínimos cuadrados de los valores de los parámetros se realiza en dos fases, una de estimación del modelo propiamente dicho y otra de escalamiento óptimo. Estas dos fases se van alternando iterativamente hasta conseguir una convergencia determinada.

La finalidad del procedimiento es conseguir cuantificaciones (puntajes) para cada una de las personas entrevistadas y, por tanto, de las categorías de las variables que sean óptimas, en el sentido de que las categorías estén separadas unas de otras en la dimensión o dimensiones estudiadas tanto como sea posible y, a su vez, dentro de cada categoría las personas estén lo más próximas unas de otras, es decir, con puntuaciones lo más homogéneas entre sí.

Una vez construido el índice de Violencia Familiar a nivel de Mujeres en edad fértil, se procedió a estimar la media de Violencia Familiar a nivel de cada una de los departamentos, para cuyo cálculo se utilizó el programa SPSS versión 15.

\section{RESULTADOS}

El Índice de Violencia Familiar a nivel país se obtuvo de los datos correspondientes a 22558 mujeres mediante la aplicación de PRINCALS en 23 reactivos correspondientes a ocho variables seleccionadas del Cuestionario ENDES continua 2007-2008. Fueron suficientes dos factores o dimensiones con Alpha de Cronbach total igual a 0,94. 
Perú: Mapa de la violencia familiar, a nivel departamental

Tabla N. ${ }^{\circ}$ 1. PERÚ: Índice global de violencia familiar e índice de violencia psicológica. 20072008.

\begin{tabular}{|c|c|c|c|c|c|c|}
\hline \multirow{2}{*}{ Índices } & \multirow{2}{*}{ Estimado } & \multirow{2}{*}{$\begin{array}{c}\text { Error } \\
\text { estándar }\end{array}$} & \multicolumn{2}{|c|}{$95 \%$ Intervalo de confianza } & \multirow{2}{*}{$\begin{array}{l}\text { Coeficiente de } \\
\text { variación }\end{array}$} & \multirow{2}{*}{$\begin{array}{c}\text { Tamaño } \\
\text { población }\end{array}$} \\
\hline & & & Bajo & Alto & & \\
\hline Índice global de violencia & 12,5794 & 0,17001 & 12,2458 & 12,913 & 0,014 & 12082,031 \\
\hline Índice de violencia psicológica & 23,4016 & 0,31627 & 22,7809 & 24,0223 & 0,014 & 12082,031 \\
\hline
\end{tabular}

Fuente: ENDES - PERÚ 2008

Elaboración: Equipo de investigación.

Según el contenido de la Tabla 01, la estimación a nivel nacional del Índice Global de Violencia Familiar (IGVF) es igual a 12,58, cuyo coeficiente de variación, 0,014, es bajo indicando que la estimación tiene baja dispersión. El Índice de Violencia Psicológica (IVP), tiene una estimación igual a 23,40 indicando que la estimación también tiene baja dispersión, con un intervalo de confianza de 22,78 a 24,02, con $95 \%$ de probabilidad de confianza. El IVP es bastante alto respecto al IGVF.

Tabla N. ${ }^{\circ}$ 2. PERÚ: Índice de Violencia Familiar según Edad. 2007-2008.

\begin{tabular}{|c|c|c|c|c|c|c|}
\hline \multirow{2}{*}{ Edad } & \multirow{2}{*}{ Estimado } & \multirow{2}{*}{$\begin{array}{l}\text { Error } \\
\text { estándar }\end{array}$} & \multicolumn{2}{|c|}{$95 \%$ Intervalo de confianza } & \multirow{2}{*}{$\begin{array}{l}\text { Coeficiente de } \\
\text { variación }\end{array}$} & \multirow{2}{*}{$\begin{array}{l}\text { Tamaño } \\
\text { muestra }\end{array}$} \\
\hline & & & Bajo & Alto & & \\
\hline 15-19 años & 11,3035 & 0,66350 & 10,0014 & 12,6056 & 0,059 & 366 \\
\hline 20-24 años & 12,1768 & 0,41198 & 11,3683 & 12,9853 & 0,034 & 1288 \\
\hline 25-29 años & 11,8436 & 0,37913 & 11,0995 & 12,5876 & 0,032 & 2159 \\
\hline 30-34 años & 12,7083 & 0,37181 & 11,9786 & 13,4379 & 0,029 & 2464 \\
\hline 35-39 años & 13,1096 & 0,39477 & 12,3348 & 13,8843 & 0,030 & 2151 \\
\hline 40-44 años & 12,9080 & 0,41636 & 12,0909 & 13,7251 & 0,032 & 1953 \\
\hline 45-49 años & 12,8589 & 0,40431 & 12,0655 & 13,6524 & 0,031 & 1700 \\
\hline
\end{tabular}

Fuente: ENDES- PERÚ 2008.

Elaboración: Equipo de investigación.

En la Tabla 2 se presenta la estimación del Índice Global de Violencia Familiar (IGVF) según grupo etarios, la mayor violencia se presenta en mujeres de 35 a 39 años (13.11), cuyo coeficiente de variación es bajo (0.030), el intervalo de confianza para el IGVF es de 12,33 a 13,88, con $95 \%$ de probabilidad de confianza. En cambio, el menor IGVF es para las edades de 15 a 19 años cuyo coeficiente de variación es bajo $(0,059)$ pero el mayor de todos, el intervalo de confianza para el IGVF, es de 10,00 a 12,61 , con $95 \%$ de probabilidad de confianza. 
Tabla N. 3. PERÚ: Índice de Violencia Familiar según Región Natural. 2007-2008.

\begin{tabular}{|c|c|c|c|c|c|c|}
\hline \multirow{2}{*}{ Región natural } & \multirow{2}{*}{ Estimado } & \multirow{2}{*}{$\begin{array}{c}\text { Error } \\
\text { estándar }\end{array}$} & \multicolumn{2}{|c|}{$95 \%$ Intervalo de confianza } & \multirow{2}{*}{$\begin{array}{l}\text { Coeficiente de } \\
\text { variación }\end{array}$} & \multirow{2}{*}{$\begin{array}{l}\text { Tamaño } \\
\text { muestra }\end{array}$} \\
\hline & & & Bajo & Alto & & \\
\hline Lima Metropolitana & 11,332 & 0,45478 & 10,4395 & 12,2244 & 0,04 & 3145 \\
\hline Resto de Costa & 11,8496 & 0,28179 & 11,2966 & 12,4026 & 0,024 & 2773 \\
\hline Sierra & 13,4823 & 0,25347 & 12,9848 & 13,9797 & 0,019 & 4605 \\
\hline Selva & 13,7274 & 0,3404 & 13,0594 & 14,3954 & 0,025 & 1559 \\
\hline
\end{tabular}

Fuente: ENDES - PERÚ 2008

Elaboración: Equipo de investigación

En la Tabla 3, se presenta la estimación del Índice Global de Violencia Familiar (IGVF) según región natural. El mayor índice estimado es para la región Selva $(13,73)$, cuyo coeficiente de variación es bajo $(0,025)$, el intervalo de confianza para el IGVF es de 13,06 a 14,40 , con $95 \%$ de probabilidad de confianza. En cambio, el menor IGVF estimado es para Lima Metropolitana (11,33), cuyo coeficiente de variación es bajo (0.04), pero el mayor de todos, el intervalo de confianza para el IGVF es de 10,44 a 12,22 , con $95 \%$ de probabilidad de confianza.

Tabla N. ${ }^{\circ}$ 4. PERÚ: Índice de Violencia Familiar según Lugar de Residencia. 2007-2008.

\begin{tabular}{lcccccc}
\hline \multirow{2}{*}{ Lugar de residencia } & Estimado & $\begin{array}{c}\text { Error } \\
\text { estándar }\end{array}$ & & $95 \%$ Intervalo de confianza & $\begin{array}{c}\text { Coeficiente de } \\
\text { variación }\end{array}$ & $\begin{array}{c}\text { Tamaño } \\
\text { muestra }\end{array}$ \\
\hline Urbano & 12,3994 & 0,21666 & 11,9742 & 12,8246 & 0,017 & 8090 \\
Rural & 12,9442 & 0,26988 & 12,4146 & 13,4739 & 0,021 & 3992 \\
\hline
\end{tabular}

Fuente: ENDES - PERÚ 2008.

Elaboración: Equipo de investigación.

En la Tabla 4 se presenta la estimación del Índice Global de Violencia Familiar (IGVF) según lugar de residencia. Muestra que el mayor índice estimado es para el área rural $(12,94)$, cuyo coeficiente de variación es bajo $(0,021)$, el intervalo de confianza para el IGVF es de 12,41 a 13,47 , con $95 \%$ de probabilidad de confianza. 
Perú: Mapa de la violencia familiar, a nivel departamental

Tabla N. ${ }^{\circ}$ 5. PERÚ: Índice global de violencia familiar, psicológica, física y sexual según área de residencia. 2007-2008.

\begin{tabular}{|c|c|c|c|c|c|c|c|}
\hline \multirow{2}{*}{ Área de residencia } & \multirow{2}{*}{ Índices } & \multirow{2}{*}{$\begin{array}{c}\text { Valor } \\
\text { estimado }\end{array}$} & \multirow{2}{*}{$\begin{array}{c}\text { Error } \\
\text { estándar }\end{array}$} & \multicolumn{2}{|c|}{$95 \%$ de intervalo de confianza } & \multirow{2}{*}{$\begin{array}{l}\text { Coeficiente } \\
\text { de variación }\end{array}$} & \multirow{2}{*}{$\begin{array}{l}\text { Número de } \\
\text { mujeres }\end{array}$} \\
\hline & & & & Inferior & Superior & & \\
\hline \multirow[t]{4}{*}{ Urbano } & IGVF & 12,40 & 0,22 & 11,97 & 12,82 & 0,02 & 8090 \\
\hline & IVP & 23,07 & 0,40 & 22,28 & 23,86 & 0,02 & 8090 \\
\hline & IVF & 25,49 & 1,07 & 23,39 & 27,59 & 0,04 & 3248 \\
\hline & IVS & 33,61 & 2,71 & 28,29 & 38,92 & 0,08 & 751 \\
\hline \multirow[t]{4}{*}{ Rural } & IGVF & 12,94 & 0,27 & 12,41 & 13,47 & 0,02 & 3992 \\
\hline & IVP & 24,08 & 0,50 & 23,10 & 25,07 & 0,02 & 3992 \\
\hline & IVF & 25,79 & 1,10 & 23,63 & 27,95 & 0,04 & 1413 \\
\hline & IVS & 46,30 & 2,71 & 40,98 & 51,62 & 0,06 & 379 \\
\hline
\end{tabular}

Fuente: ENDES - PERÚ 2008

Elaboración: Equipo de Investigación.

En la Tabla 5 se presenta la estimación del Índice Global de Violencia Familiar (IGVF), el Îndice de Violencia Psicológica (IVP), el Î́ndice de Violencia Física (IVF), y el Î́ndice de Violencia Sexual (IVS), según área de residencia, muestran que en el área de residencia Urbano, el de mayor valor es el IVS, sin embargo el IVP $(23,07)$, cuyo coeficiente de variación es bajo $(0,02)$, el intervalo de confianza para el IVP es de 22,28 a 23,86, con $95 \%$ de probabilidad de confianza. En el área de residencia Rural, el de mayor valor es el IVS, sin embargo el IVP $(24,08)$, cuyo coeficiente de variación es bajo $(0,02)$, el intervalo de confianza para el IVP es de 23,10 a 25,07 con $95 \%$ de probabilidad de confianza.

Tabla N. ${ }^{\circ}$ 6. PERÚ: Índice global de violencia familiar, psicológica, física y sexual según estado civil. 2007-2008.

\begin{tabular}{|c|c|c|c|c|c|c|c|}
\hline \multirow{2}{*}{ Estado civil } & \multirow{2}{*}{ Índices } & \multirow{2}{*}{$\begin{array}{c}\text { Valor } \\
\text { estimado }\end{array}$} & \multirow{2}{*}{$\begin{array}{l}\text { Error } \\
\text { estándar }\end{array}$} & \multicolumn{2}{|c|}{$95 \%$ de intervalo de confianza } & \multirow{2}{*}{$\begin{array}{l}\text { Coeficiente } \\
\text { de variación }\end{array}$} & \multirow{2}{*}{$\begin{array}{l}\text { Número de } \\
\text { mujeres }\end{array}$} \\
\hline & & & & Inferior & Superior & & \\
\hline \multirow{4}{*}{$\begin{array}{l}\text { Casada o } \\
\text { conviviente }\end{array}$} & IGVF & 11,36 & 0,17 & 11,02 & 11,70 & 0,02 & 10505 \\
\hline & IVP & 21,13 & 0,32 & 20,49 & 21,76 & 0,02 & 10505 \\
\hline & IVF & 28,08 & 0,87 & 26,37 & 29,78 & 0,03 & 3708 \\
\hline & IVS & 46,95 & 2,53 & 41,98 & 51,92 & 0,05 & 777 \\
\hline \multirow{4}{*}{$\begin{array}{l}\text { Divorciada / } \\
\text { viuda / separada }\end{array}$} & IGVF & 20,73 & 0,56 & 19,63 & 21,82 & 0,03 & 1577 \\
\hline & IVP & 38,56 & 1,04 & 36,51 & 40,60 & 0,03 & 1577 \\
\hline & IVF & 15,87 & 1,41 & 13,10 & 18,63 & 0,09 & 953 \\
\hline & IVS & 17,91 & 2,87 & 12,27 & 23,55 & 0,16 & 353 \\
\hline
\end{tabular}

Fuente: ENDES - PERÚ 2008.

Elaboración: Equipo de investigación. 
En la Tabla 6 se presenta la estimación del Índice Global de Violencia Familiar (IGVF), el Índice de Violencia Psicológica (IVP), el Índice de Violencia Física (IVF), y el Índice de Violencia Sexual (IVS), según estado civil, revelan que en el grupo de casada/conviviente, el índice de mayor valor es el IVS, sin embargo el IVP $(21,13)$ cuyo coeficiente de variación es bajo $(0,02)$, el intervalo de confianza para el IVP es de 20,49 a 21,76 , con $95 \%$ de probabilidad de confianza. En el otro grupo, el de mayor valor es el IVP $(38,56)$ cuyo coeficiente de variación es bajo $(0,03)$, el intervalo de confianza para el IVP es de 36,51 a 40,60 con $95 \%$ de probabilidad de confianza.

Tabla N. ${ }^{\circ}$ 7. PERÚ: Índice global de violencia familiar por departamentos, 2007-2008.

\begin{tabular}{|c|c|c|c|c|c|c|c|}
\hline \multirow{2}{*}{ Departamento } & \multirow{2}{*}{$\begin{array}{c}\text { Valor } \\
\text { estimado }\end{array}$} & \multirow{2}{*}{$\begin{array}{c}\text { Error } \\
\text { estándar }\end{array}$} & \multicolumn{2}{|c|}{$95 \%$ de intervalo de confianza } & \multirow{2}{*}{$\begin{array}{l}\text { Coeficiente } \\
\text { de variación }\end{array}$} & \multicolumn{2}{|c|}{ Número de mujeres } \\
\hline & & & Inferior & Superior & & Ponderados & Sin ponderar \\
\hline Total & 12,58 & 0,17 & 12,25 & 12,91 & $\mathbf{0 , 0 1}$ & 12082 & 12568 \\
\hline Loreto & 16,13 & 0,65 & 14,86 & 17,40 & 0,04 & 364 & 408 \\
\hline Apurímac & 15,80 & 1,09 & 13,66 & 17,93 & 0,07 & 197 & 409 \\
\hline Pasco & 14,67 & 0,76 & 13,19 & 16,15 & 0,05 & 118 & 570 \\
\hline Madre de Dios & 14,60 & 0,59 & 13,45 & 15,75 & 0,04 & 55 & 754 \\
\hline Arequipa & 14,28 & 0,66 & 12,99 & 15,57 & 0,05 & 494 & 630 \\
\hline Puno & 14,06 & 0,68 & 12,74 & 15,39 & 0,05 & 717 & 445 \\
\hline Huancavelica & 14,01 & 1,04 & 11,96 & 16,06 & 0,07 & 233 & 400 \\
\hline Cusco & 13,98 & 1,12 & 11,78 & 16,18 & 0,08 & 521 & 343 \\
\hline Junín & 13,59 & 0,70 & 12,21 & 14,98 & 0,05 & 522 & 340 \\
\hline Ayacucho & 13,44 & 0,78 & 11,90 & 14,98 & 0,06 & 331 & 518 \\
\hline Ancash & 13,38 & 0,92 & 11,58 & 15,18 & 0,07 & 447 & 447 \\
\hline Huánuco & 13,24 & 0,90 & 11,47 & 15,01 & 0,07 & 364 & 386 \\
\hline La Libertad & 13,16 & 0,57 & 12,03 & 14,28 & 0,04 & 700 & 410 \\
\hline Tumbes & 13,09 & 0,61 & 11,89 & 14,29 & 0,05 & 105 & 679 \\
\hline San Martín & 13,02 & 0,56 & 11,92 & 14,11 & 0,04 & 355 & 643 \\
\hline Tacna & 12,91 & 0,50 & 11,93 & 13,89 & 0,04 & 161 & 501 \\
\hline Moquegua & 12,72 & 0,59 & 11,57 & 13,88 & 0,05 & 85 & 564 \\
\hline Ucayali & 12,72 & 0,50 & 11,74 & 13,69 & 0,04 & 190 & 658 \\
\hline Piura & 12,07 & 0,65 & 10,79 & 13,34 & 0,05 & 738 & 504 \\
\hline Amazonas & 11,57 & 0.82 & 9,95 & 13,18 & 0,07 & 203 & 471 \\
\hline Ica & 11,38 & 0,44 & 10,53 & 12,24 & 0,04 & 338 & 563 \\
\hline Lima & 11,36 & 0,42 & 10,54 & 12,17 & 0,04 & 3606 & 877 \\
\hline Cajamarca & 11,31 & 0,54 & 10,26 & 12,37 & 0,05 & 776 & 509 \\
\hline Lambayeque & 10,43 & 0,50 & 9,44 & 11,42 & 0,05 & 463 & 539 \\
\hline
\end{tabular}

Fuente: ENDES - PERÚ 2008.

Elaboración: Equipo de investigación. 


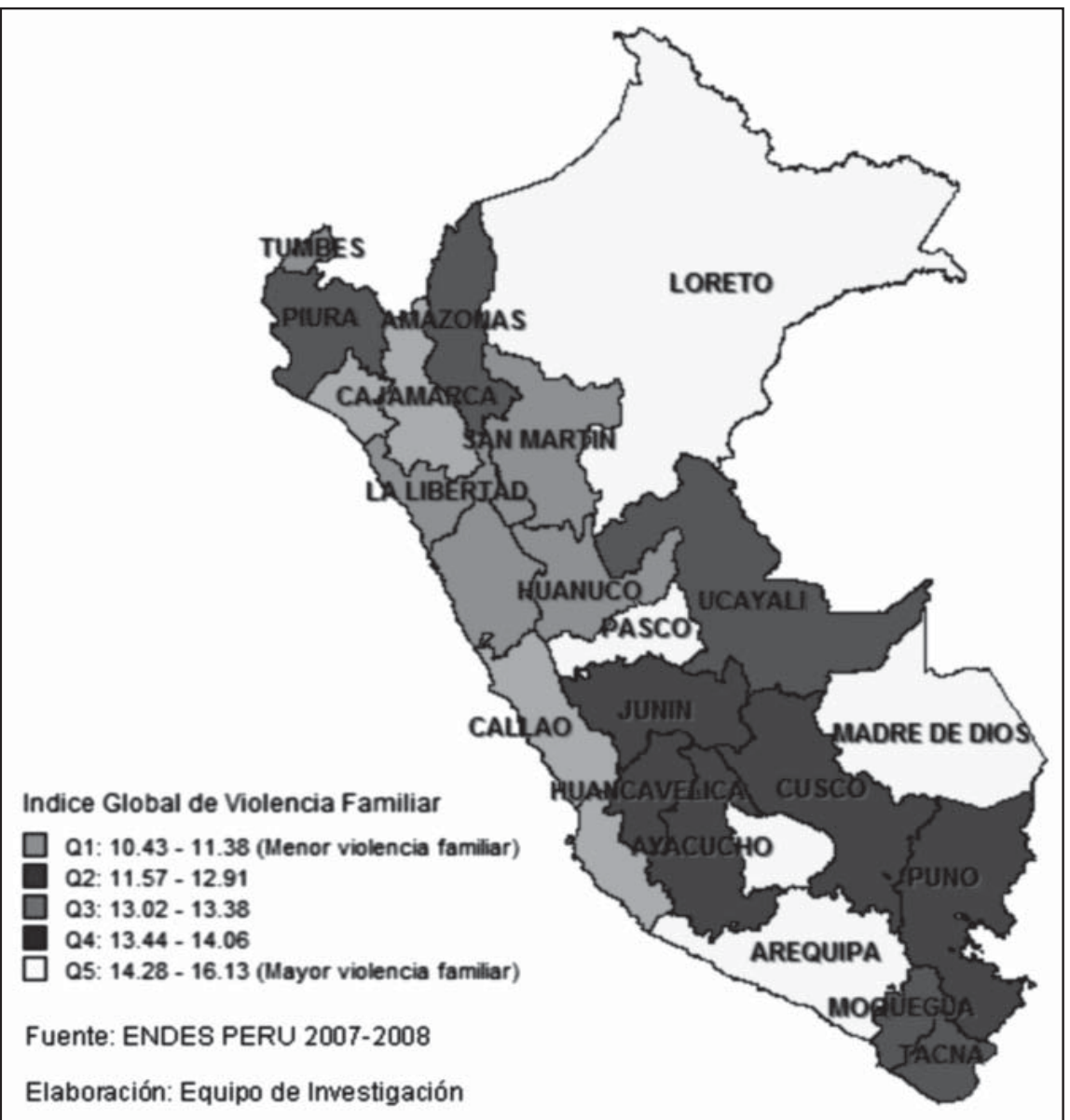

Figura N. ${ }^{\circ}$ 1. Mapa del índice global de violencia familiar a nivel departamental, 2008.

En la Tabla 7 y Figura 1 se presenta la estimación del Índice Global de Violencia Familiar (IGVF) por departamentos, para una mejor comprensión se han dividido en quintos. Los departamentos están ordenados de manera decreciente, el primer grupo está integrado por los departamentos: Loreto, Apurímac, Pasco, Madre de Dios y Arequipa; departamentos que conforman el $20 \%$ de aquellos con mayor IGVF que fluctúa entre 14,28 y 16,13. El $20 \%$ de departamentos con IGVF más bajos, cuyos valores fluctúan entre 10,43 y 13,38 está constituido por Ica, Lima, Cajamarca y Lambayeque.

\section{DISCUSIÓN}

El alto relieve que alcanza el Índice de Violencia Psicológica (IVP) $(23,4016)$ en comparación con el índice Global de Violencia (IGV) (12,5794), según la Tabla 1, tiene, entre otras, las siguientes implicancias: 
1. Este hecho concuerda con lo hallado por otros investigadores (Hurtado y Jaffe, 1990; Gallardo, J.A. y Jiménez, M, 1997), según los cuales, los niños al ser testigos de la agresión física cruel y reiterada del padre o padrastro contra la madre (testigos impotentes de poder defenderla) tiene propiedades mayores y más perniciosamente traumatizantes que el recibir tales agresiones físicas (violencia física). Lo expresado configura violencia psicológica que sufren los niños en referencia.

En el mismo sentido, Arias (1999) sostiene que el abuso psicológico (Violencia psicológica) posee mayor poder predictivo respecto al desarrollo del Trastorno de estrés post traumático que la severidad o frecuencia del daño físico recibido (Violencia física).

2. El hallazgo de que el grupo etario femenino de 35 a 39 años presenta el mayor Índice Global de Violencia (IGV) (Tabla 2), guarda armonía con lo constatado por Famularo (1999) en sus estudios realizados en este campo, según los cuales las mujeres, en su mayoría, tardan 10 años en pedir ayuda, debido, probablemente, a temores a las consecuencias personales, familiares y sociales que puedan sobrevenir a dicho pedido.

3. La violencia sexual presenta acentuado predominio en el ámbito rural frente al ámbito urbano (IGV: 46,30 frente a 33,61, respectivamente, según la Tabla 4). Este hecho otorga fuerte respaldo empírico a lo hallado por Labrador (2004), en el sentido de que las niñas que son víctimas de abuso sexual presentan mayor riesgo de sufrir victimización sexual y traumatismo post traumático en la edad adulta. Schaff (1998) encontró en sus investigaciones resultados análogos. La premisa implícita en esta interpretación es que la violencia sexual que afecta fuertemente a la mujer en todas sus edades, en el ámbito rural, configura procesos cíclicos y recurrentes que se expresan en el índice de violencia sexual.

\section{CONCLUSIONES}

1. Los resultados muestran que en el Perú de hoy, la violencia familiar, en sus diferentes formas (violencia física, violencia psicológica y violencia sexual) está extendida y arraigada en todo el territorio y distribuida con ciertas diferencias a nivel departamental.

2. La violencia familiar está estrechamente asociada a factores culturales ancestrales, como mitos y creencias que se transmiten de generación en generación, cultura de la violencia, machismo, especialmente en los agresores, y pasividad de la mujer, todo ello, exacerbado por la pobreza (Miljanovich et al., 2009). Esto último suele provocar serias distorsiones en la dinámica familiar y favorecer la tendencia a la perpetuación del citado tipo de violencia.

3. La diferenciación de la violencia familiar según departamentos requiere ser estudiada interdisciplinariamente, en busca de constructos explicativos que, además, favorezcan las aplicaciones de los resultados, en cuanto a promoción de la salud integral, con énfasis en la salud mental y las respectivas acciones de prevención. 
Perú: Mapa de la violencia familiar, a nivel departamental

\section{NOTA DE RECONOCIMIENTO}

Al Consejo de Gestión de la Investigación. Vicerectorado de Investigación de la Universidad Nacional Mayor de San Marcos.

Al Licenciado en Estadística Fernando Camones Gonzales por su apoyo técnico y profesional.

\section{REFERENCIAS BIBLIOGRÁFICAS}

1. Bardales M. Olga y Huallpa A. Elisa (2005). Maltrato y Abuso Sexual en Niñas, Niños y Adolescentes: Estudio realizado en los distritos de San Martín de Porras, Cuzco e Iquitos. Lima: MINDES Programa Nacional contra la Violencia Familiar y Sexual.

2. Bell, Richard (2004). Lecture 8: Categorical Data Analysis 1: Optimal Scaling. Australia: University of Melbourne. Disponible en: http://www.psych.unimelb. edu.au/staff/bell.html/.

3. Bigman, D. and H. Fofack (2000). Geographical Targeting for Poverty Alleviation: An Introduction to the Special Issue. The World Bank Economic Review 14 (1):129-145.

4. Escobal, Javier; Torero, Máximo y Ponce, Carmen (2001). "Focalización Geográfica del Gasto Social: Mapas de Pobreza". Red CIES de Pobreza GRAD. APOYO Informe Final. [Visto 03 nov 2010]. Disponible en: http://cies.org.pe/ files/active/0/mapas.pdf

5. Guezmes, A. Palomino, N. y Ramos, M. (2002). Violencia sexual y física contra las mujeres en el Perú. Estudio multicéntrico de la OMS sobre la violencia de pareja y la salud de las mujeres. Lima.

6. Klevens Joanne (2001). Violencia física contra la mujer en Santa Fe de Bogotá: prevalencia y factores asociados. Rev. Panam. Salud Pública [serial on the Internet] [cited 2010 Dec 08]; 9(2): 78-83. Available from: http://www.scielosp.org/scielo. php? script $=$ sci_arttext\&pid $=$ S1020-49892001000200004\&lng $=$ en. doi: $10.1590 /$ S1020-49892001000200004.

7. Labrador, F.J. y Rincón, P.P. (2002). Trastorno de estrés postraumático en víctimas de maltrato doméstico: evaluación de un programa de intervención. Análisis y Modificación de Conducta, 28, 905-932.

8. Labrador, F.J.; Rincón, P.P.; De Luis, P. y Fernánde.Velasco, R. (2004). Mujeres víctimas de la violencia doméstica. Madrid: Pirámide.

9. Mardia K. V.; Kent J. T.; Bibby J. M. (1979). Multivariate analysis. New York: Academic Press.

10. Miljanovich M. et al. (2010). Exposición Taller Avances de Investigaciones sobre Violencia Familiar. UNMSM. Vicerrectorado Académico. 
11. Meulmann, J. y Heiser Willem, J. (2001). SPSS Categories 11.0 - Manual del SPSS. Chicago: SPSS Inc.

12. Naciones Unidas. Centro de Documentación. [Visto 03 nov 2010]. Disponible en: http://freeofviolence.org/campaig-spanish/internationalday.htm

13. Programa Nacional contra la Violencia Familiar y Sexual. Ministerio de la Mujer. [Acceso nov 2010]. Disponible en: http://www.mimdes.gob.pe/estadisticas-pncvfs. html

14. Ramos Padilla, Miguel Ángel (2003). Violencia sexual y física contra las mujeres adolescentes y jóvenes del Perú. Ponencia presentada en el II Congreso Internacional de Salud y Desarrollo de los adolescentes y Jóvenes. Organizado por la Sociedad Peruana de Adolescencia y Juventud. Del 13 al 16 de Mayo 2003. Lima, Perú.

15. Ravallion, M. y Q. Woodon (1997). "Poor areas or only poor people?". Policy Research Working Paper 1798. World Bank, Washington.

16. Viviano, Teresa (2007). ¿Qué son los centros Emergencia Mujer?: Situación actual y perspectivas dentro del proceso de descentralización. Lima: Industrias Gráficas Ausangraf.

17. Young, Forrest W. (1981). Quantitative Analysis of Qualitative Data. Psychometrika, Vol 46, N. ${ }^{\circ}$ 4, december, 1981.

18. Young, F. W., Tanake Y. y De Leeuw J. (1978). The Principal Components of Mixed Measurement Level Multivariate Data: Alternating Least Squares Method With Optimal Scaling Features. Psychometrika, Vol 43, N. ${ }^{\circ}$ 2, junio). 